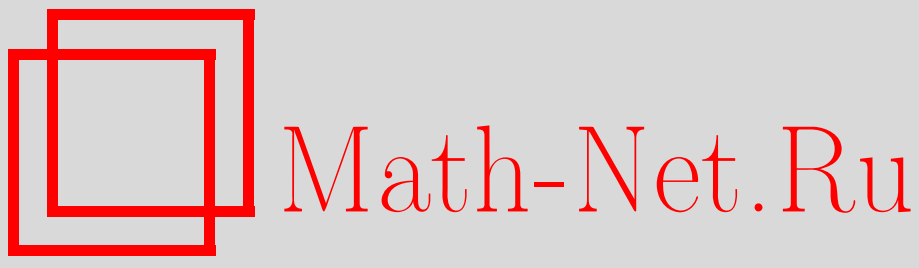

А. Р. Данилин, Аппроксимация сингулярно возмущенной эллиптической задачи оптимального управления, Матем. сб̆., 2000, том 191, номер 10, 3-12

DOI: https://doi.org/10.4213/sm512

Использование Общероссийского математического портала Math-Net.Ru подразумевает, что вы прочитали и согласны с пользовательским соглашением

http://www . mathnet.ru/rus/agreement

Параметры загрузки:

IP: 3.80 .181 .102

26 апреля 2023 г., 02:11:18 


\section{А.Р. Данилин}

\section{Аппроксимация сингулярно возмущенной эллиптической задачи оптимального управления}

\footnotetext{
Рассматривается задача оптимального управления решениями эллиптического уравнения с мальм параметром при старших производных в прямоугольнике, две стороны которого параллельны характеристике предельного уравнения. Найдена пределная задача и получены асимптотические оценки для решений задачи, аппроксимирующей исходную.

Библиограбия: 11 названий.
}

В работе рассматривается одна задача Ж.-Л. Лионса [1] оптимального управления решениями уравнения эллиптического типа с малым коэффициентом при старшей производной в области с частью границы, параллельной характеристике. Найдена предельная задача и получены асимптотические оценки для решений задачи, аппроксимируюшей исходную. Другие сингулярные задачи оптимального управления решениями эллиптических уравнений рассматривались в [2], [3]. Сингулярные задачи для уравнения эллиптического типа с малым коэффициентом при старшей производной в области с частью границы, параллельной характеристике, рассматривались также в [4]-[6].

Примеры оптимальных задач для уравнений и систем уравнений в частных производных, в частности, эллиптического типа, дают механика сплошной среды, оптимизация элементов конструкций и т. п. [7], что говорит о важности изучения различных аспектов таких задач.

1. Постановка задачи и основные соотношения. В области $\Omega:=(0 ; 1) \times$ $(0 ; 1) \subset \mathbb{R}^{2}$ рассматривается следующая задача оптимального управления (см. $[1 ;$ гл. $2, \S 2$, соотношения $(2.8),(2.9)])$ :

$$
\begin{gathered}
\mathscr{L}_{\varepsilon} z(x, y)=f(x, y)-u(x, y), \quad(x, y) \in \Omega, \quad z \in H_{0}^{1}(\Omega), \\
J_{\nu}(u):=\int_{\Omega}\left(z^{2}(x, y)+\nu \cdot u^{2}(x, y)\right) d x d y \rightarrow \inf , \quad u \in \mathscr{U} .
\end{gathered}
$$

Здесь $\nu>0, H_{0}^{1}(\Omega)$ - соболевское пространство функций, равных нулю на границе $\partial \Omega$,

$$
\begin{gathered}
\mathscr{L}_{\varepsilon} z:=\varepsilon^{2} \Delta z-b(x) \frac{\partial z}{\partial y}-a(x, y) z, \\
f, a \in C^{\infty}(\bar{\Omega}), \quad a(x, y) \geqslant A>0 \text { при } \quad(x, y) \in \Omega, \\
b \in C^{\infty}([0,1]), \quad b(x) \geqslant B>0 \text { при } \quad x \in[0,1], \\
\mathscr{U}:=\left\{u(\cdot) \in L_{2}(\Omega):\|u\|^{2}:=\int_{\Omega} u^{2}(x, y) d x d y \leqslant R^{2}\right\} .
\end{gathered}
$$

Работа вьполнена при поддержке Российского фонда фундаментальных исследований (гранты №№ 96-15-96241, 99-01-00139).

(C) А. Р. ДАнилин 2000 
В этом случае единственное оптимальное управление $u_{\varepsilon}(\cdot)$ и соответствующее ему $z_{\varepsilon}(\cdot)$ находятся как решение следуюшей задачи (см. [1; гл. $2, \S 2$, соотношения $(2.10)],[3])$ :

$$
\begin{aligned}
& \mathscr{L}_{\varepsilon} z_{\varepsilon}+u_{\varepsilon}=f, \quad \mathscr{L}_{\varepsilon}^{*} u_{\varepsilon}-\lambda_{\varepsilon} \cdot z_{\varepsilon}=0, \quad z_{\varepsilon}, u_{\varepsilon} \in H_{0}^{1}(\Omega), \\
& \left\|u_{\varepsilon}\right\| \leqslant R, \quad\left(\frac{1}{\nu}-\lambda_{\varepsilon}\right) \cdot\left(R-\left\|u_{\varepsilon}\right\|\right)=0, \quad 0<\lambda_{\varepsilon} \leqslant \frac{1}{\nu}
\end{aligned}
$$

где

$$
\mathscr{L}_{\varepsilon}^{*} u:=\varepsilon^{2} \Delta u+b(x) \frac{\partial u}{\partial y}-a(x, y) u .
$$

Отметим, что в силу $(1) z_{\varepsilon}, u_{\varepsilon} \in C^{\infty}(\Omega)$. Это следует из того, что в силу первого эллиптического уравнения из (2) порядок гладкости $z_{\varepsilon}$ на две единицы больше, чем у $u_{\varepsilon}$, а в силу второго - наоборот, порядок гладкости $u_{\varepsilon}$ на две единицы больше, чем у $z_{\varepsilon}$.

2. Предельная задача. Рассмотрим вопрос о поведении $\lambda_{\varepsilon}, z_{\varepsilon}$ и $u_{\varepsilon}$ при $\varepsilon \rightarrow 0$. Предельной задачей для (2) будет задача

$$
\begin{cases}\mathscr{L}_{0} z:=-b(x) \frac{\partial z}{\partial y}-a(x, y) z=f-u, & z(x, 0)=0, \\ \mathscr{L}_{0}^{*} u:=b(x) \frac{\partial u}{\partial y}-a(x, y) u=\lambda z, & u(x, 1)=0, \\ \left(\frac{1}{\nu}-\lambda\right) \cdot(R-\|u\|)=0, & \|u\| \leqslant R, \quad 0<\lambda \leqslant \frac{1}{\nu} .\end{cases}
$$

ПРЕДЛОЖЕНИЕ 1. В классе непрерывных функиий задача

$$
\mathscr{L}_{0} z+u=f, \quad \mathscr{L}_{0}^{*} u-\lambda z=g, \quad z(x, 0)=\varphi(x), \quad u(x, 1)=\psi(x)
$$

разрешима единственным образом при любых $\lambda \in \mathbb{R}, f, g \in C(\bar{\Omega})$ u $\varphi, \psi \in$ $C([0,1])$.

ДокАЗАТЕльство. Задача (5) эквивалентна системе интегральных уравнений с непрерывными ядрами. Поскольку интегральные операторы с непрерывными ядрами на компактах являются вполне непрерывными операторами, то согласно первой альтернативе Фредгольма достаточно проверить единственность решения задачи с $f=g=0$ и $\varphi=\psi=0$. Пусть $\mathscr{L}_{0} z+u=0, \mathscr{L}_{0}^{*} u-\lambda z=0, z(x, 0)=0$, $u(x, 1)=0$. Тогда, умножив первое уравнение на $u$ и проинтегрировав его по $[0,1]$, получим

$$
\begin{aligned}
0 & =\int_{0}^{1} u^{2}(x, y) d y+\int_{0}^{1} \mathscr{L}_{0} z(x, y) \cdot u(x, y) d y \\
& =\int_{0}^{1} u^{2}(x, y) d y+\int_{0}^{1} z(x, y) \cdot \mathscr{L}_{0}^{*}(x, y) d y \\
& =\int_{0}^{1} u^{2}(x, y) d y+\lambda \cdot \int_{0}^{1} z^{2}(x, y) d y .
\end{aligned}
$$

В силу непрерьвности функций $u$ и $z$ это означает, что $u=z=0$.

Отметим также, что

$$
\text { если } z(x, 0) \equiv 0 \equiv u(x, 1), \quad \text { то } \int_{0}^{1} \mathscr{L}_{0} z \cdot u d y \equiv \int_{0}^{1} z \cdot \mathscr{L}_{0}^{*} u d y .
$$


ПРЕДЛОЖЕНИЕ 2 . Пусть $z(\cdot ; \lambda)$ и и(·; $\lambda)$ - решение краевой задачи из (4). Ecлu

$$
\mathscr{L}_{0}^{*} f=b(x) \frac{\partial f(x, y)}{\partial y}-a(x, y) \cdot f(x, y) \not \equiv 0 \quad \text { в } \quad \Omega,
$$

то функция $h(\lambda):=\|u(\cdot ; \lambda)\|^{2}$ строго возрастает и $h(0)=0$.

ДокАЗАТЕльство. Покажем, что $h^{\prime}(\lambda)>0$ при $\lambda>0$. Так как

$$
h^{\prime}(\lambda)=2\left(u(\cdot ; \lambda), \frac{\partial}{\partial \lambda} u(\cdot ; \lambda)\right):=\int_{\Omega} u(x, y ; \lambda) \cdot \frac{\partial}{\partial \lambda} u(x, y ; \lambda) d x d y,
$$

то рассмотрим подробнее функции $w(\cdot):=\frac{\partial}{\partial \lambda} z(\cdot ; \lambda)$ и $v(\cdot):=\frac{\partial}{\partial \lambda} u(\cdot ; \lambda)$.

В силу теоремы о дифференцируемости решений системы обькновенных дифференциальных уравнений $w$ и $v$ удовлетворяют соотношениям

$$
\mathscr{L}_{0} w+v=0, \quad \mathscr{L}_{0}^{*} v-\lambda w=z(\cdot ; \lambda), \quad w(x, 0)=0, \quad v(x, 1)=0 .
$$

Пусть $\lambda>0$. Положим $V(x, y):=v(x, y)-\frac{1}{\lambda} u(x, y)$. Тогда $V(x, 1)=0, u(x, y ; \lambda)=$ $\lambda \cdot v(x, y)-\lambda \cdot V(x, y)$ и

$$
\mathscr{L}_{0}^{*} V=\mathscr{L}_{0}^{*} v-\frac{1}{\lambda} \mathscr{L}_{0}^{*} u(\cdot ; \lambda)=z(\cdot ; \lambda)+\lambda w-\frac{1}{\lambda} \cdot \lambda z(\cdot ; \lambda),
$$

т.е.

$$
\mathscr{L}_{0}^{*} V=\lambda \cdot w .
$$

Поэтому

$$
\begin{aligned}
h^{\prime}(\lambda) & =2(u(\cdot ; \lambda), v)=(\lambda v-\lambda V, v) \stackrel{(9)}{=} \lambda\|v\|^{2}-\lambda\left(V,-\mathscr{L}_{0} w\right) \\
& \stackrel{(6)}{=} \lambda\|v\|^{2}+\lambda\left(\mathscr{L}_{0}^{*} V, w\right) \stackrel{(10)}{\geqslant} 0 .
\end{aligned}
$$

Если $h^{\prime}(\lambda)=0$, то $v=0=w$, что в силу $(9)$ дает $z(\cdot ; \lambda)=0$. Но тогда $f=u(\cdot ; \lambda)$ и $\mathscr{L}_{0}^{*} f \equiv 0$ в $\Omega$, что противоречит условию $(7)$.

В дальнейшем считается выполненьм следуюшее неравенство

$$
h\left(\frac{1}{\nu}\right)>R .
$$

Тогда в условиях предложения 2 задача (4) имеет единственное решение $z_{0}$, $u_{0}, \lambda_{0}$, причем

$$
0<\lambda_{0}<\frac{1}{\nu}, \quad\left\|u_{0}\right\|=R \quad \text { и } z_{0} \not \equiv 0 .
$$

Вопрос о поведении $z_{\varepsilon}, u_{\varepsilon}$, и $\lambda_{\varepsilon}$ решает следуюшая 
ТЕОРемА 1. При выполнении условий (7) и (11)

$$
\lambda_{\varepsilon} \rightarrow \lambda_{0}, \quad z_{\varepsilon} \underset{L_{2}(\Omega)}{\longrightarrow} z_{0}, \quad u_{\varepsilon} \underset{L_{2}(\Omega)}{\longrightarrow} u_{0} \quad n p u \quad \varepsilon \rightarrow 0
$$

где $\lambda_{0}, z_{0}$ и и $u_{0}$ - единственное решение задачи (4) с таким $\lambda_{0}$, что $\left\|u_{0}\right\|=R$. $\Pi$ При этом $\left\|u_{\varepsilon}\right\|=R$ для всех достаточно мальх $\varepsilon>0$.

ДоКАЗАТЕльство. Предположим противное. Тогда найдутся последовательность $\{\varepsilon(n)\}, \varepsilon(n) \rightarrow 0$, и $\alpha>0$ такие, что

$$
|| z_{n}-z_{0}\|+\| u_{n}-u_{0} \|+\left|\lambda_{n}-\lambda_{0}\right| \geqslant \alpha
$$

где $\lambda_{n}:=\lambda_{\varepsilon(n)}, z_{n}:=z_{\varepsilon(n)}, u_{n}:=u_{\varepsilon(n)}$. Поскольку последовательность $\left\{\lambda_{n}\right\}$ ограничена, то можно считать, что

$$
\lambda_{n} \rightarrow \bar{\lambda} \in\left[0, \frac{1}{\nu}\right]
$$


лу Грина и учитьвая, что

$$
\begin{aligned}
\left(\frac{\partial}{\partial y} z_{n}, z_{n}\right) & =\int_{\Omega} \frac{\partial}{\partial y} z_{n}(x, y) \cdot z_{n}(x, y) d x d y \\
& =\left.\frac{1}{2} \int_{0}^{1} z_{n}^{2}(x, y)\right|_{y=0} ^{y=1} d x=0=\left(\frac{\partial}{\partial y} u_{n}, u_{n}\right)
\end{aligned}
$$

получим

$$
\begin{gathered}
\left\|\sqrt{a} \cdot z_{n}\right\|^{2}+\left\|\nabla \varepsilon(n) z_{n}\right\|^{2}=\left(u_{n}-f, z_{n}\right), \\
\left\|\sqrt{a} \cdot u_{n}\right\|^{2}+\left\|\nabla \varepsilon(n) u_{n}\right\|^{2}=\lambda_{n}\left(z_{n}, u_{n}\right) .
\end{gathered}
$$

Тогда $A\left\|z_{n}\right\|^{2} \leqslant\left\|\sqrt{a} \cdot z_{n}\right\|^{2} \leqslant\left\|u_{n}-f\right\| \cdot\left\|z_{n}\right\|$, что с учетом ограниченности последовательности $\left\{u_{n}\right\}$ в $L_{2}(\Omega)$ дает ограниченность последовательности $\left\{z_{n}\right\}$ в $L_{2}(\Omega)$. Это, в свою очередь, влечет ограниченность $\left\{\nabla \varepsilon(n) z_{n}\right\}$ и $\left\{\nabla \varepsilon(n) u_{n}\right\}$ в $L_{2}(\Omega)$. Таким образом, последовательности $\left\{\varepsilon(n) z_{n}\right\}$ и $\left\{\varepsilon(n) u_{n}\right\}$ ограничены в $H_{0}^{1}(\Omega)$ и можно считать, что $\varepsilon(n) z_{n} \underset{H_{0}^{1}(\Omega)}{\stackrel{\text { сл. }}{\longrightarrow}} \widetilde{z}$ и $\varepsilon(n) u_{n} \underset{H_{0}^{1}(\Omega)}{\stackrel{\text { сл. }}{\longrightarrow}} \widetilde{u}$. В силу компактности вложения $H_{0}^{1}(\Omega)$ в $L_{2}(\Omega)$ получим: $\varepsilon(n) z_{n} \underset{L_{2}(\Omega)}{\longrightarrow} \widetilde{z}$ и $\varepsilon(n) u_{n} \underset{L_{2}(\Omega)}{\longrightarrow} \widetilde{u}$, что вместе с ограниченностью последовательностей $\left\{z_{n}\right\}$ и $\left\{u_{n}\right\}$ дает соотношения

$$
\varepsilon(n) z_{n} \underset{H_{0}^{1}(\Omega)}{\stackrel{\text { сл. }}{\longrightarrow}} 0 \text { и } \varepsilon(n) u_{n} \underset{H_{0}^{1}(\Omega)}{\stackrel{\text { сл. }}{\longrightarrow}} 0 .
$$

Пусть $\bar{z}$ и $\bar{u}$ - решение краевой задачи из (4) при $\lambda=\bar{\lambda}$, а

$$
\bar{z}_{m}:=\bar{z}(x, y) \cdot \sigma_{m}(x), \quad \bar{u}_{m}:=\bar{u}(x, y) \cdot \sigma_{m}(x)
$$


где $\sigma_{m}(x)$ - “срезающая" функция такая, что

$$
\sigma_{m}(x)=\left\{\begin{array}{l}
1, \frac{1}{m}<x<1-\frac{1}{m} \\
0, x<\frac{1}{2 m} \text { или } x>1-\frac{1}{2 m} .
\end{array}\right.
$$

Тогда

$$
\left\|\bar{z}_{m}\right\| \leqslant\|\bar{z}\|, \quad\left\|\bar{u}_{m}\right\| \leqslant\|\bar{u}\|, \quad \bar{z}_{m} \underset{L_{2}(\Omega)}{\longrightarrow} \bar{z}, \quad \bar{u}_{m} \underset{L_{2}(\Omega)}{\longrightarrow} \bar{u} .
$$

Отметим, что $\bar{z}_{m}(0, y)=\bar{z}_{m}(1, y)=\bar{u}_{m}(0, y)=\bar{u}_{m}(1, y)=0$ и

$$
\begin{aligned}
\mathscr{L}_{0} \bar{z}_{m}+\bar{u}_{m} & =f \cdot \sigma_{m}(x), & & \mathscr{L}_{0}^{*} \bar{u}_{m}-\bar{\lambda} \cdot \bar{z}_{m}=0, \\
\bar{z}_{m}(x, 0) & =0, & & \bar{u}_{m}(x, 1)=0 .
\end{aligned}
$$

Пусть $z_{n, m}:=z_{n}-\bar{z}_{m}, u_{n, m}:=u_{n}-\bar{u}_{m}, \lambda_{n, m}:=\lambda_{n}-\bar{\lambda}_{m}$. Тогда

$$
\begin{aligned}
\mathscr{L}_{n} z_{n, m}+u_{n, m} & =\mathscr{L}_{n} z_{n}-\varepsilon^{2}(n) \Delta \bar{z}_{m}-\mathscr{L}_{0} \bar{z}_{m}+u_{n}-\bar{u}_{m} \\
& \stackrel{(2),(18)}{=} f-\varepsilon^{2}(n) \Delta \bar{z}_{m}-f \cdot \sigma_{m}, \\
\mathscr{L}_{n}^{*} u_{n, m}-\lambda_{n} \cdot z_{n, m} & =\mathscr{L}_{n}^{*} u_{n}-\varepsilon^{2}(n) \Delta \bar{u}_{m}-\mathscr{L}_{0}^{*} \bar{u}_{m}-\lambda_{n} \cdot z_{n}+\lambda_{n} \cdot \bar{z}_{m} \\
& =-\varepsilon^{2}(n) \Delta \bar{u}_{m}-\bar{\lambda} \cdot \bar{z}_{m}+\bar{\lambda}_{n} \cdot \bar{z}_{m} .
\end{aligned}
$$

Итак,

$$
\begin{aligned}
\mathscr{L}_{n} z_{n, m}+u_{n, m} & =-\varepsilon^{2}(n) \Delta \bar{z}_{m}-f_{m}, \\
\mathscr{L}_{n}^{*} u_{n, m}-\lambda_{n} \cdot z_{n, m} & =-\varepsilon^{2}(n) \Delta \bar{u}_{m}-\bar{\lambda}_{n} \cdot \bar{z}_{m},
\end{aligned}
$$

где $f_{m}(x, y):=f(x, y) \cdot\left(1-\sigma_{m}(x)\right) \underset{L_{2}(\Omega)}{\longrightarrow} 0$.

Рассмотрим величину

$$
\begin{aligned}
\left(\mathscr{L}_{n} z_{n, m}, u_{n, m}\right)-\left(z_{n, m}, \mathscr{L}_{n}^{*} u_{n, m}\right)=\left(\mathscr{L}_{n} z_{n}-\varepsilon^{2}(n) \Delta \bar{z}_{m}-\mathscr{L}_{0} \bar{z}_{m}, u_{n}-\bar{u}_{m}\right) & \\
& -\left(z_{n}-\bar{z}_{m}, \mathscr{L}_{n}^{*} u_{n}-\varepsilon^{2}(n) \Delta \bar{u}_{m}-\mathscr{L}_{0}^{*} \bar{u}_{m}\right) \\
= & \left(\mathscr{L}_{n} z_{n}, u_{n}\right)-\left(\mathscr{L}_{n} z_{n}, \bar{u}_{m}\right)-\varepsilon^{2}(n)\left(\Delta \bar{z}_{m}, u_{n}\right)+\varepsilon^{2}(n)\left(\Delta \bar{z}_{m}, \bar{u}_{m}\right) \\
& -\left(\mathscr{L}_{0} \bar{z}_{m}, u_{n}\right)+\left(\mathscr{L}_{0} \bar{z}_{m}, \bar{u}_{m}\right)-\left(z_{n}, \mathscr{L}_{n}^{*} u_{n}\right)+\varepsilon^{2}(n)\left(z_{n}, \Delta \bar{u}_{m}\right) \\
& +\left(z_{n}, \mathscr{L}_{0}^{*} \bar{u}_{m}\right)+\left(\bar{z}_{m}, \mathscr{L}_{n}^{*} u_{n}\right)-\varepsilon^{2}(n)\left(z_{m}, \Delta \bar{u}_{m}\right)-\left(\bar{z}_{m}, \mathscr{L}_{0}^{*} \bar{u}_{m}\right) \\
= & -\varepsilon^{2}(n)\left(\Delta z_{n}, \bar{u}_{m}\right)-\left(\mathscr{L}_{0} z_{n}, \bar{u}_{m}\right)-\left(\mathscr{L}_{0} \bar{z}_{m}, u_{n}\right)+\left(z_{n}, \mathscr{L}_{0}^{*} \bar{u}_{m}\right) \\
& +\varepsilon^{2}(n)\left(\bar{z}_{m}, \Delta u_{n}\right)+\left(\bar{z}_{m}, \mathscr{L}_{0}^{*} u_{n}\right)+\bar{\gamma}_{n, m} \\
= & \bar{\gamma}_{n, m}-\varepsilon^{2}(n)\left(\Delta z_{n}, \bar{u}_{m}\right)+\varepsilon^{2}(n)\left(\bar{z}_{m}, \Delta u_{n}\right),
\end{aligned}
$$

где $\bar{\gamma}_{n, m} \rightarrow 0$ при $n \rightarrow \infty$ и любом фиксированном $m$.

Покажем, что $\varepsilon^{2}(n)\left(\Delta z_{n}, \bar{u}_{m}\right) \rightarrow 0$ и $\varepsilon^{2}(n)\left(\bar{z}_{m}, \Delta u_{n}\right) \rightarrow 0$ при $n \rightarrow \infty$. Имеем

$$
\varepsilon^{2}(n)\left(\Delta z_{n}, \bar{u}_{m}\right)=-\varepsilon^{2}(n)\left(\nabla z_{n}, \nabla u_{m}\right)-\varepsilon^{2}(n) \int_{0}^{1} \bar{u}_{m}(x, 0) \cdot \frac{\partial}{\partial y} z_{n}(x, 0) d x .
$$

Первое слагаемое в (20) стремится к нулю при $n \rightarrow \infty$ в силу (16). Рассмотрим второе слагаемое. 
Поскольку при $v \in H_{0}^{1}(\Omega)$ имеет место соотношение

$$
\varepsilon^{2}(n)\left(\Delta z_{n}, v\right) \stackrel{(16)}{=} \varepsilon^{2}(n)\left(\nabla z_{n}, \nabla v\right) \rightarrow 0 \text { при } n \rightarrow \infty
$$

то последовательность $\left\{\varepsilon^{2}(n) \Delta z_{n}\right\}$ поточечно ограничена в $H_{0}^{1}(\Omega)$ как последовательность линейных непрерывных функционалов. Поэтому по теореме Банаха-Штейнгауза [8] $\left\{\varepsilon^{2}(n) \Delta z_{n}\right\}$ ограничена в $H_{0}^{1}(\Omega)$ и, следовательно, в $L_{2}(\Omega)$. Аналогично показывается ограниченность в $L_{2}(\Omega)$ последовательностей $\left\{\varepsilon^{2}(n) \Delta u_{n}\right\},\left\{\varepsilon^{2}(n) \frac{\partial^{2}}{\partial x^{2}} z_{n}\right\}$ и $\left\{\varepsilon^{2}(n) \frac{\partial^{2}}{\partial x^{2}} u_{n}\right\}$. Таким образом, в силу (2) последовательности $\left\{\frac{\partial}{\partial y} z_{n}\right\}$ и $\left\{\frac{\partial}{\partial y} u_{n}\right\}$ ограничены в $L_{2}(\Omega)$.

Будем рассматривать первое уравнение системы (2) как обыкновенное дифференциальное уравнение, зависящее от параметра $x$ :

$$
\varepsilon^{2}(n) \frac{\partial^{2}}{\partial y^{2}} z_{n}-b(x) \frac{\partial}{\partial y} z_{n}=g_{n}(x, y):=a(x, y)-u_{n}+f
$$

где последовательность $\left\{g_{n}\right\}$ ограничена в $L_{2}(\Omega)$. Тогда

$$
\frac{\partial}{\partial y} z_{n}=C_{n}(x) \cdot \exp \left(\frac{b(x) \cdot y}{\varepsilon^{2}(n)}\right)+\frac{1}{\varepsilon^{2}(n)} \int_{1}^{y} \exp \left(\frac{b(x)(y-\bar{y})}{\varepsilon^{2}(n)}\right) \cdot g(x, \bar{y}) d \bar{y} .
$$

Поэтому последовательность $\left\{\varepsilon^{2}(n) C_{n}(x) \cdot \exp \left(b(x) \cdot y / \varepsilon^{2}(n)\right)\right\}$ ограничена в $L_{2}(\Omega)$, т.e.

$$
\begin{aligned}
K & \geqslant \varepsilon^{4}(n) \int_{0}^{1} d x \int_{0}^{1} C_{n}^{2}(x) \cdot \exp \left(\frac{2 b(x) \cdot y}{\varepsilon^{2}(n)}\right) d y \\
& =\frac{\varepsilon^{2}(n)}{2} \int_{0}^{1} \frac{C_{n}^{2}(x)}{b(x)} \cdot\left(\exp \left(\frac{2 b(x)}{\varepsilon^{2}(n)}-1\right)\right) d x \\
& \geqslant \frac{\varepsilon^{2}(n)}{2}\left(\max _{0 \leqslant x \leqslant 1} b(x)\right)^{-1} \cdot\left(\exp \left(\frac{2 B}{\varepsilon^{2}(n)}-1\right)\right) \cdot \int_{0}^{1} C_{n}^{2}(x) d x \\
& =\varepsilon^{2}(n) \cdot K_{2} \cdot\left(\exp \left(\frac{2 B}{\varepsilon^{2}(n)}-1\right)\right) \cdot \int_{0}^{1} C_{n}^{2}(x) d x .
\end{aligned}
$$

Таким образом,

$$
\int_{0}^{1} C_{n}^{2}(x) d x \leqslant K_{3} \cdot \varepsilon^{-2}(n) \cdot\left(\exp \left(\frac{2 B}{\varepsilon^{2}(n)}-1\right)\right)^{-1} .
$$

Оценим второе слагаемое в (20):

$$
\begin{gathered}
\left|\varepsilon^{2}(n) \int_{0}^{1} u_{m}(x, 0) \cdot \frac{\partial}{\partial y} z_{n}(x, 0) d x\right| \stackrel{(21)}{\leqslant} \varepsilon^{2}(n)\left|\int_{0}^{1} C_{n}(x) \cdot u_{m}(x, 0) d x\right| \\
+\left|\int_{0}^{1} u_{m}(x, 0) \cdot\left(\int_{y}^{1} \exp \left(\frac{b(x)(y-\bar{y})}{\varepsilon^{2}(n)}\right) \cdot g_{n}(x, \bar{y}) d \bar{y}\right) d x\right| .
\end{gathered}
$$


Ho

$$
\begin{aligned}
\varepsilon^{2}(n)\left|\int_{0}^{1} C_{n}(x) \cdot u_{m}(x, 0) d x\right| & \leqslant \varepsilon^{2}(n)\left(\int_{0}^{1} C_{n}^{2}(x) d x\right)^{1 / 2} \cdot\left(\int_{0}^{1} u_{m}^{2}(x, 0) d x\right)^{1 / 2} \\
& \stackrel{(22)}{\leqslant} K_{4} \cdot \varepsilon(n) \cdot\left(\exp \left(\frac{2 B}{\varepsilon^{2}(n)}-1\right)\right)^{-1 / 2} \rightarrow 0
\end{aligned}
$$

при $n \rightarrow \infty$. Аналогично

$$
\begin{aligned}
& \left|\int_{0}^{1} u_{m}(x, 0) \cdot\left(\int_{y}^{1} \exp \left(\frac{b(x)(y-\bar{y})}{\varepsilon^{2}(n)}\right) \cdot g(x, \bar{y}) d \bar{y}\right) d x\right| \\
& \quad \leqslant K_{5} \cdot \varepsilon(n) \rightarrow 0 \text { при } n \rightarrow 0 .
\end{aligned}
$$

Таким образом, в силу $(20) \varepsilon^{2}(n) \cdot\left(\Delta z_{n}, \bar{u}_{m}\right) \rightarrow 0$ при $n \rightarrow \infty$. Аналогично показывается соотношение $\varepsilon^{2}(n) \cdot\left(\bar{z}_{m}, \Delta u_{n}\right) \rightarrow 0$ при $n \rightarrow \infty$. Итак,

$$
\left(\mathscr{L}_{n} z_{n, m}, u_{n, m}\right)=\left(z_{n, m}, \mathscr{L}_{n}^{*} u_{n, m}\right)+\bar{\gamma}_{n, m} \text { и } \bar{\gamma}_{n, m} \rightarrow 0 \text { при } n \rightarrow \infty .
$$

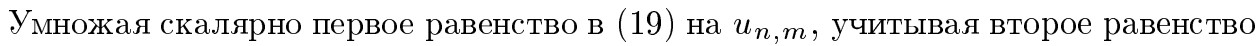
этой системы и (24), получим

$$
\begin{aligned}
& \left(f_{m}, u_{n, m}\right)-\varepsilon^{2}(n)\left(\Delta \bar{z}_{m}, u_{n, m}\right)=\left\|u_{n, m}\right\|^{2}+\left(\mathscr{L}_{n} z_{n, m}, u_{n, m}\right) \\
& \quad=\left\|u_{n, m}\right\|^{2}+\left(z_{n, m}, \mathscr{L}_{n}^{*} u_{n, m}\right)+\bar{\gamma}_{n, m} \\
& \quad=\left\|u_{n, m}\right\|^{2}+\lambda_{n} \cdot\left\|z_{n, m}\right\|^{2}-\varepsilon^{2}(n)\left(\Delta \bar{u}_{m}, z_{n, m}\right)+\bar{\lambda}_{n} \cdot\left(\bar{z}_{m}, z_{n, m}\right),
\end{aligned}
$$

т.е.

$$
\left\|u_{n, m}\right\|^{2}+\lambda_{n} \cdot\left\|z_{n, m}\right\|^{2}=\gamma_{n, m} \rightarrow 0 \text { при } n \rightarrow \infty .
$$

Так как $\gamma_{n, m} \rightarrow 0$ при $n \rightarrow \infty$ и каждом фиксированном $m$, то найдется последовательность $\{n(m)\}: n(m) \rightarrow \infty, \gamma_{n(m), m} \rightarrow 0$ при $m \rightarrow \infty$. Тогда из соотношения (25) следует, что

$$
u_{n(m), m}=u_{n(m)}-\bar{u}_{m} \rightarrow 0, \quad \lambda_{n(m), m} \cdot z_{n(m), m}=\lambda_{n(m), m} \cdot\left(z_{n(m)}-\bar{z}_{m}\right) \rightarrow 0
$$

при $m \rightarrow \infty$ в $L_{2}(\Omega)$. Это вместе с (14) и (17) дает

$$
u_{n(m)} \rightarrow \bar{u} \text { и } \lambda_{n(m), m} \cdot \bar{z}_{m} \rightarrow \bar{\lambda} \cdot \bar{z} \text { при } m \rightarrow \infty \text { в } L_{2}(\Omega) .
$$

Таким образом, $\left\|u_{n(m), m}\right\| \rightarrow\|\bar{u}\|$. Перейдем в равенстве

$$
\left(\frac{1}{\nu}-\lambda_{n(m)}\right) \cdot\left(R-\left\|u_{n(m)}\right\|\right)=0
$$

к пределу при $m \rightarrow \infty$. Получим

$$
\left(\frac{1}{\nu}-\bar{\lambda}\right) \cdot(R-\|\bar{u}\|)=0 .
$$

Если $1 / \nu=\bar{\lambda}$, то $R<\|\bar{u}\|$, что противоречит неравенствам $R \geqslant\left\|u_{n(m)}\right\|$. Поэтому $1 / \nu>\bar{\lambda} \geqslant 0$ и $R=\|\bar{u}\|$. Тогда $\|\bar{u}\| \neq 0$ и по предложению 2 выполняется соотношение $\bar{\lambda} \neq 0$, что в силу (26) дает $z_{n(m)} \rightarrow \bar{z}$ в $L_{2}(\Omega)$. С учетом единственности решения задачи (4) получим, что $\bar{\lambda}=\lambda_{0}, \bar{z}=z_{0}$, а $\bar{u}=u_{0}$. Но это противоречит соотношению (13). Итак, $\lambda_{\varepsilon} \rightarrow \lambda_{0}, z_{\varepsilon} \underset{L_{2}(\Omega)}{\longrightarrow} z_{0}, u_{\varepsilon} \underset{L_{2}(\Omega)}{\longrightarrow} u_{0}$ при $\varepsilon \rightarrow 0$. При этом так как $\lambda_{\varepsilon} \rightarrow \lambda_{0}<1 / \nu$, то и при всех достаточно малых $\varepsilon>0$ справедливо неравенство $\lambda_{\varepsilon}<1 / \nu$ и, следовательно, $\left\|u_{\varepsilon}\right\|=R$. 
3. Аппроксимационные свойства исходной задачи. В силу теоремы 1 исходная задача эквивалентна задаче Дирихле для системы, зависящей от параметра $\lambda_{\varepsilon}$ :

$$
\begin{cases}\mathscr{L} z_{\varepsilon}+u_{\varepsilon}=f, \quad \mathscr{L}^{*} u_{\varepsilon}-\lambda_{\varepsilon} y_{\varepsilon}=0, & (x, y) \in \Omega, \\ z_{\varepsilon}(x, y)=u_{\varepsilon}(x, y)=0, & (x, y) \in \partial \Omega,\end{cases}
$$

с дополнительным условием

$$
\left\|u_{\varepsilon}\right\|^{2}=\int_{\Omega} u_{\varepsilon}^{2}(x, y) d x d y=R^{2}
$$

ТЕОРЕМа 2. Пусть выполнены условия теоремы 1 и для некоторого $\alpha>0$ функиии $Z_{\varepsilon}, U_{\varepsilon} \in C^{\infty}(\Omega)$ и $\Lambda_{\varepsilon}$ удовлетворяют следующим соотношениям:

$$
\begin{cases}\mathscr{L} Z_{\varepsilon}+U_{\varepsilon}-f=o\left(\varepsilon^{\alpha}\right), \quad \mathscr{L}^{*} U_{\varepsilon}-\Lambda_{\varepsilon} Z_{\varepsilon}=o\left(\varepsilon^{\alpha}\right), & (x, y) \in \Omega \\ Z_{\varepsilon}(x, y)=o\left(\varepsilon^{\alpha}\right), \quad U_{\varepsilon}(x, y)=o\left(\varepsilon^{\alpha}\right), & (x, y) \in \partial \Omega \\ \left\|U_{\varepsilon}\right\|=R+o\left(\varepsilon^{\alpha}\right) & \end{cases}
$$

при $\varepsilon \rightarrow 0$ в смысле метрики пространств $C^{2}(\Omega), C^{2}(\partial \Omega)$ соответственно, $a \Lambda_{\varepsilon}-\lambda_{0}=o(1), Z_{\varepsilon}(x)-z_{0}(x)=o(1), U_{\varepsilon}(x)-u_{0}(x)=o(1) n p u \varepsilon \rightarrow 0$ в $L_{2}(\Omega)$.

Тогда $\Lambda_{\varepsilon}-\lambda_{\varepsilon}=o\left(\varepsilon^{\alpha}\right), Z_{\varepsilon}-z_{\varepsilon}=o\left(\varepsilon^{\alpha-1}\right), U_{\varepsilon}-u_{\varepsilon}=o\left(\varepsilon^{\alpha-1}\right) n p u \varepsilon \rightarrow 0$ в $C(\bar{\Omega})$, где $z_{\varepsilon}, u_{\varepsilon}$ и $\lambda_{\varepsilon}-$ решение задачи (2).

ДокАЗАТЕльство. Пусть $\bar{z}:=Z_{\varepsilon}-z_{\varepsilon}, \bar{u}:=U_{\varepsilon}-u_{\varepsilon}$ и $\bar{\lambda}:=\lambda^{\varepsilon}-\lambda_{\varepsilon}$. Тогда в силу условий теоремы и соотношений (27)

$$
\begin{cases}\mathscr{L} \bar{z}+\bar{u}=o\left(\varepsilon^{\alpha}\right), \quad \mathscr{L}^{*} \bar{u}-\Lambda_{\varepsilon} \bar{z}-\bar{\lambda} Z_{\varepsilon}=o\left(\varepsilon^{\alpha}\right), & (x, y) \in \Omega \\ \bar{z}=o\left(\varepsilon^{\alpha}\right), \quad \bar{u}=o\left(\varepsilon^{\alpha}\right), & (x, y) \in \partial \Omega \\ \left(u_{\varepsilon}, \bar{u}\right)=-\frac{1}{2}\|\bar{u}\|^{2}+o\left(\varepsilon^{\alpha}\right) & \end{cases}
$$

и по теореме $1 \bar{z} \underset{L_{2}(\Omega)}{\longrightarrow} 0, \bar{u} \underset{L_{2}(\Omega)}{\longrightarrow} 0$ и $\bar{\lambda} z \rightarrow 0$ при $\varepsilon \rightarrow 0$.

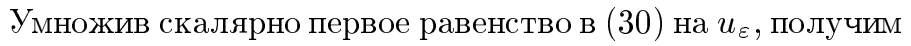

$$
\begin{aligned}
\left(\mathscr{L}_{\varepsilon} \bar{z}, u_{\varepsilon}\right)+\left(\bar{u}, u_{\varepsilon}\right) \stackrel{\text { ф. }}{\stackrel{\Gamma \text { рина }}{=}}\left(\bar{z}, \mathscr{L}_{\varepsilon}^{*} u_{\varepsilon}\right)+o\left(\varepsilon^{\alpha}\right)+\left(\bar{u}, u_{\varepsilon}\right) \\
\stackrel{(27)}{=} \lambda_{\varepsilon} \cdot\left(\bar{z}, z_{\varepsilon}\right)+\left(\bar{u}, u_{\varepsilon}\right)+o\left(\varepsilon^{\alpha}\right)=o\left(\varepsilon^{\alpha}\right)
\end{aligned}
$$

т.e.

$$
\lambda_{\varepsilon} \cdot\left(\bar{z}, z_{\varepsilon}\right)+\left(\bar{u}, u_{\varepsilon}\right)=o\left(\varepsilon^{\alpha}\right) .
$$

Аналогично с учетом равенства (31) после умножения на $z_{\varepsilon}$ получим

$$
\begin{aligned}
\left(\mathscr{L}_{\varepsilon}^{*} \bar{u}, z_{\varepsilon}\right)- & \lambda_{\varepsilon}\left(\bar{z}, z_{\varepsilon}\right)-\bar{\lambda}\left(Z_{\varepsilon}, z_{\varepsilon}\right) \\
\text { ф. }{ }^{\Gamma \text { pина }} & \left(\bar{u}, \mathscr{L}_{\varepsilon} z_{\varepsilon}\right)-\lambda_{\varepsilon}\left(\bar{z}, z_{\varepsilon}\right)-\bar{\lambda}\left(Z_{\varepsilon}, z_{\varepsilon}\right)+o\left(\varepsilon^{\alpha}\right) \\
& \stackrel{(27)}{=}(\bar{u}, f)-\left(\bar{u}, u_{\varepsilon}\right)-\lambda_{\varepsilon}\left(\bar{z}, z_{\varepsilon}\right)-\bar{\lambda}\left(Z_{\varepsilon}, z_{\varepsilon}\right)+o\left(\varepsilon^{\alpha}\right) \\
& \stackrel{(31)}{=}(\bar{u}, f)-\bar{\lambda}\left(Z_{\varepsilon}, z_{\varepsilon}\right)+o\left(\varepsilon^{\alpha}\right) \stackrel{(30)}{=} o\left(\varepsilon^{\alpha}\right),
\end{aligned}
$$


или

$$
(\bar{u}, f)=\bar{\lambda}\left(Z_{\varepsilon}, z_{\varepsilon}\right)+o\left(\varepsilon^{\alpha}\right) .
$$

Наконец, умножив второе равенство в (30) скалярно на $\bar{z}$, получим

$$
\begin{aligned}
& \left(\mathscr{L}_{\varepsilon}^{*} \bar{u}, \bar{z}\right)-\lambda_{\varepsilon} \cdot\|\bar{z}\|^{2}-\bar{\lambda}\left(Z_{\varepsilon}, \bar{z}\right) \\
& \stackrel{{ }^{\text {риина }}}{=}\left(\bar{u}, \mathscr{L}_{\varepsilon} \bar{z}\right)+o\left(\varepsilon^{2 \alpha}\right)-\lambda_{\varepsilon} \cdot\|\bar{z}\|^{2}-\bar{\lambda}\left(Z_{\varepsilon}, \bar{z}\right) \\
& \stackrel{(30))}{=}-\|\bar{u}\|^{2}+o\left(\varepsilon^{\alpha}\right) \cdot\|\bar{u}\|+o\left(\varepsilon^{2 \alpha}\right)-\lambda_{\varepsilon} \cdot\|\bar{z}\|^{2}-\bar{\lambda}\left(\|\bar{z}\|^{2}+\left(Z_{\varepsilon}, \bar{z}\right)\right) \\
& \quad-\|\bar{u}\|^{2}+o\left(\varepsilon^{\alpha}\right) \cdot\|\bar{u}\|+o\left(\varepsilon^{2 \alpha}\right)-\Lambda_{\varepsilon} \cdot\|\bar{z}\|^{2}-\bar{\lambda} \cdot\left(Z_{\varepsilon}, \bar{z}\right) \\
& \stackrel{(30),(31)}{=}-\|\bar{u}\|^{2}+o\left(\varepsilon^{\alpha}\right) \cdot\|\bar{u}\|+o\left(\varepsilon^{2 \alpha}\right)-\frac{\bar{\lambda}}{\lambda_{\varepsilon}} \cdot\left(\frac{1}{2}\|\bar{u}\|^{2}+o\left(\varepsilon^{\alpha}\right)\right)+o\left(\varepsilon^{2 \alpha}\right) \\
& \quad o\left(\varepsilon^{\alpha}\right) \cdot\|\bar{z}\| .
\end{aligned}
$$

Но из $(32)$ и того, что $\left(\bar{z}, z_{\varepsilon}\right) \rightarrow\left\|z_{0}\right\|^{2} \neq 0$ (см. (12)), следует, что

$$
\bar{\lambda}=\|\bar{u}\| \cdot O(1)+o\left(\varepsilon^{\alpha}\right) .
$$

Поэтому

$$
-\|\bar{u}\|^{2} \cdot\left(1+\frac{\bar{\lambda}}{2 \lambda_{\varepsilon}}\right)-\lambda_{\varepsilon} \cdot\|\bar{z}\|^{2}+o\left(\varepsilon^{\alpha}\right) \cdot\|\bar{u}\|-\frac{1}{\lambda_{\varepsilon}}\|\bar{u}\| \cdot o\left(\varepsilon^{\alpha}\right)+o\left(\varepsilon^{2 \alpha}\right)=o\left(\varepsilon^{\alpha}\right) \cdot\|\bar{z}\|,
$$

или

$$
\|\bar{u}\|^{2} \cdot\left(1+\frac{\bar{\lambda}}{2 \lambda_{\varepsilon}}\right)+\Lambda_{\varepsilon} \cdot\|\bar{z}\|^{2}=o\left(\varepsilon^{\alpha}\right) \cdot(\|\bar{z}\|+\|\bar{u}\|)+o\left(\varepsilon^{2 \alpha}\right)
$$

Но $\|\bar{z}\|+\|\bar{u}\| \asymp\left(\|\bar{z}\|^{2}+\|\bar{u}\|^{2}\right)^{1 / 2}$, а в силу того, что $\bar{\lambda} \rightarrow 0, \Lambda_{\varepsilon} \rightarrow \lambda_{0} \neq 0$, справедливо соотношение

$$
\|\bar{u}\|^{2} \cdot\left(1+\frac{\bar{\lambda}}{2 \lambda_{\varepsilon}}\right)+\Lambda_{\varepsilon} \cdot\|\bar{z}\|^{2} \asymp\|\bar{z}\|^{2}+\|\bar{u}\|^{2}
$$

поэтому $\|\bar{z}\|^{2}+\|\bar{u}\|^{2}=o\left(\varepsilon^{\alpha}\right) \cdot\left(\|\bar{z}\|^{2}+\|\bar{u}\|^{2}\right)^{1 / 2}+o\left(\varepsilon^{2 \alpha}\right)$. Таким образом, $\|\bar{z}\|+$ $\|\bar{u}\|=o\left(\varepsilon^{\alpha}\right)$ и, тем самым, $\|\bar{z}\|=o\left(\varepsilon^{\alpha}\right)$ и $\|\bar{u}\|=o\left(\varepsilon^{\alpha}\right)$. Формула (33) показьвает, что и $\bar{\lambda}=o\left(\varepsilon^{\alpha}\right)$.

Теперь, умножив первое уравнение в (30) на $\bar{z}$, получим

$$
(\bar{u}, \bar{z})+\left(\mathscr{L}_{\varepsilon} \bar{z}, \bar{z}\right)=o\left(\varepsilon^{2 \alpha}\right)+\varepsilon^{2} \cdot\|\nabla \bar{z}\|^{2}-(a \cdot \bar{z}, \bar{z})=o\left(\varepsilon^{\alpha}\right) \cdot\|\bar{z}\|,
$$

или $\|\nabla \bar{z}\|=o\left(\varepsilon^{\alpha-1}\right)$. Аналогично и $\|\nabla \bar{u}\|=o\left(\varepsilon^{\alpha-1}\right)$. Таким образом, $\|\bar{z}\|_{H^{1}(\Omega)}=$ $o\left(\varepsilon^{\alpha-1}\right)$ и $\|\bar{u}\|_{H^{1}(\Omega)}=o\left(\varepsilon^{\alpha-1}\right)$. Но $\bar{z}, \bar{u} \in C^{2}(\Omega)$, поэтому в силу формулы $(5.3)$ из [9]

$$
\|\bar{z}\|_{H^{2}(\Omega)} \leqslant K \cdot\left(\|\bar{z}\|_{H^{1}(\Omega)}+\left\|\mathscr{L}_{\varepsilon} \bar{z}\right\|_{L_{2}(\Omega)}+\left\|o\left(\varepsilon^{\alpha}\right)-\bar{z}\right\|_{H^{2}(\partial \Omega)}\right)=o\left(\varepsilon^{\alpha-1}\right),
$$

что в силу теоремы вложения $[10]$ дает $\|\bar{z}\|_{C(\bar{\Omega})}=o\left(\varepsilon^{\alpha-1}\right)$. Аналогично и $\|\bar{z}\|_{C(\bar{\Omega})}=$ $o\left(\varepsilon^{\alpha-1}\right)$. 
Теорема 2 показывает, что для построения асимптотики функций $z_{\varepsilon}, u_{\varepsilon}$ и $\lambda_{\varepsilon}$ достаточно построить функции $Z_{\varepsilon}, U_{\varepsilon}$ и $\Lambda_{\varepsilon}$, удовлетворяющие соотношениям (29).

Функции $Z_{\varepsilon}, U_{\varepsilon}$ и $\Lambda_{\varepsilon}$ можно определить с помощью частичных сумм согласованных асимптотических рядов [11], построенных для различных подобластей исходного квадрата. Такое построение в силу нарастания степенных особенностей у внешнего разложения в окрестностях угловых точек существенно сложнее ситуаций, возникающих для одного эллиптического уравнения или системы, связанной обшими граничными условиями [5]-[7].

Построение полного асимптотического разложения, позволяющего аппроксимировать $z_{\varepsilon}, u_{\varepsilon}, \lambda_{\varepsilon}$ в $\bar{\Omega}$ с точностью до любой степени малого параметра $\varepsilon$, предполагается опубликовать позднее.

\section{Список литературы}

1. Лионс ЖК.-Л. Оптимальное управление системами, описьваемыми уравнениями с частными производными. М.: Мир, 1972.

2. Данилин А. Р. Асимптотика ограниченных управлений для сингулярной эллиптической задачи в области с малой полостью // Матем. сб. 1998. Т. 189. № 11. С. 27-60.

3. Kaпустян B. E. Асимптотика ограниченных управлений в оптимальных эллиптических задачах // Докл. АН Украины. Сер. математика, естествознание, технические науки. 1992. № 2. C. 70-74.

4. Ильин А. М., Леликова Е.Ф. Асимптотика решений некоторых эллиптических уравнений в неограниченных областях // Матем. сб. 1982. Т. 119 (161). № 3. С. 307-324.

5. Леликова E. $\Phi$. Метод сращивания асимптотических разложений для уравнения $\varepsilon \Delta u-a u_{z}=f$ в параллелепипеде // Дифференц. уравнения. 1978. Т. 14. № 9 . C. $1638-1648$.

6. Калякин Л.А. Асимптотика решения системы двух линейных уравнений МГД с сингулярньм возмущением. І. Стандартная задача в эллиптическом слое // Дифференц. уравнения. 1982. Т. 18. № 10. С. 1724-1738.

7. Литвинов В. Г. Оптимизация в эллиптических граничных задачах с приложениями к механике. М.: Наука, 1987.

8. Banach S., Steinhaus H. Sur le principle de la condensation de singularités // Fund. Math. 1927. V. 9. P. 50-61.

9. Лионс Ж.-Л., Мадженес Э. Неоднородные граничные задачи и их приложения. М.: Мир, 1971.

10. Соболев С. Л. Некоторые применения функционального анализа в математической физике. Л.: Изд-во ЛГУ, 1950.

11. Ильин A. М. Согласование асимтотических разложений решений краевых задач. М.: Наука, 1989.

Институт математики и механики УрО РАН

Поступила в редакцию

E-mail : dar@imm.uran.ru 31.05 .1999 и 30.12 .1999 